\title{
Therapy of Hyponatremia: End of Era or Minority Report?
}

\author{
Ahmed N Ghanem* \\ Consultant Urologist Surgeon, Egypt
}

Received: 鴊: November 21, 2018; Published: 制: December 03, 2018

*Corresponding author: Ahmed N Ghanem, Consultant Urologist Surgeon No1 President Mubarak Street, Mansoura 35511, Egypt

Abbreviations: SS: Sheehan Syndrome; TURP: Transurethral Resection of the Prostate; ADH: Anti-Diuretic Hormone; ARF: Acute Renal Failure; VO: Volumetric Overload; HST: Hypertonic Sodium Therapy

\section{Introduction}

I congratulate the authors on their success in investigating and treating this case of severe hyponatremia (HN) [BMJ 2008;337: a2377]. I admire the impressive style and delightfully stimulating "Endgame" analysis. The report highlighted hormonal pathways that play vital role in the patho-physiology and replacement/ maintenance therapy of secondary HN of Sheehan Syndrome (SS). The case report seems impeccable but appraisal/criticism using scientific reasoning analysis illustrates the multidimensional complexity of HN puzzle. It demonstrates how the primary insult causing SS and its secondary HN remains obscure that may be a different primary cause of HN. In sport spirit, may I contribute thoughts to this intellectual "Endgame" analysis? I have spent my career life seriously studying, analysing and resolving the puzzle of HN starting with that of the transurethral resection of the prostate (TURP) syndrome [1] and beyond [2]. The first reported case of $\mathrm{HN}$ as the TURP syndrome [3] was made possible after serum sodium estimation became available in clinical practice- as based on previous original animal research work [4].

Brainstorming and anecdotal therapy of HN was first aired at the BMJ and Lancet when I had nothing but observations, ideas and questions. Now I have nothing but answers believed to be worth more than singing about in my kitchen! The points mentioned below are all relevant to the diagnosis, primary insult, pathoaetiology, clinical presentation and the correct therapy of HN.

I. The report mentioned the diagnosis based on timely detection of HN Nadir of $116 \mathrm{mmol} / \mathrm{l}$. It occurred postoperatively- 2 days before acute presentation with cerebral MASKs that may range from confusion to coma [5] while its earlier hyper-acute presentation MASK is mainly circulatory shock [6]. Other hyper-acute presentation MASKs include severe cardiac [7], respiratory [8] and renal failure or sudden arrest and death.

II. The condition presenting with shock MASK to urologists, gynecologist, surgeons and anesthetists in theatre and ICU earlier, is the same as that presenting, with coma MASK to physicians later. Its main serum marker is the HN Nadir, if detected.

III. The original event of difficult labour, ruptured uterus, blood loss (of 1.5L in this case), and caesarean section surgery are important predisposing factors for the insult inducing brain and pituitary edema, infarction and later atrophy.

IV. The pituitary oedema is part of a general oedema affecting brain cells among other vital organs but the brain is contained within the skull. The pituitary is perhaps more vulnerable to vascular necrosis being contained within its closed Selal Turcica. This is a compartmental compression syndrome that compromises its blood supply causing infarction, so it may be the only affected but other cerebral areas and cranial nerves have been reported.

V. The use of vasopressin or ergometrine recommended for control of uterine bleeding [8] in the developed countries and, at times, incorrectly used to induce labour in developing countries, plus the endogenous vasopressin or anti-diuretic hormone $(\mathrm{ADH})$ release are also important predisposing factors in the etiology of primary HN causing brain/pituitary oedema and SS.

VI. The quantity of 5\% Dextrose solution given during labour and surgery should be mentioned, as it augments the primary 
dilution HN causing brain cell oedema and/or pituitary oedema that cause SS and its secondary HN. In lethal cases, 6-7l of 5\% Dextrose were reported, by a great author and authority on HN [9] with a correct hypothesis of cellular hypoxia-anoxia [9], despite normal arterial oxygen saturation.

VII. The mentioned hypotension "episode" is consistent primary insult of both the TURP syndrome [6] and SS, that is usually but incorrectly attributed to a known type of shock such as hemorrhagic, hypovolemic or septic shocks.

VIII. The quantity and type of fluids used for combating this hypotension "episode" of shock, to induce volume expansion and to correct hypotension are relevant to the pathogenesis of the brain/pituitary oedema. Please compare the quantity of fluid used to correct a hypotension episode with a 1.51 of blood loss.

IX. Based on volume and osmotic milieu regulations, therapeutic fluids used in clinical practice are of two types: Sodium-free fluids (type 1) and sodium-based fluids (type 2) that induce volumetric overload (VO) with respect to time (t) of the types; V01 and V02, , respectively [1].

$\mathrm{X}$. Such volume/time (VO/) concept is directly responsible yet took years to unravel as it is rarely reported or thought relevant as it conflicts with received wisdom.

XI. The classification and type of HN of both primary and secondary to SS, in relation to clinical and biochemical severity grade, require 2 more grades worse than severe in prognosis to represent cases of "critical" and "close" to death cases [5-12].

XII. The induction rate, period to presentation and correction rate signify time importance.

XIII. Grading of severity should match the HN nadir, clinical presentation and the VO/t gain.

XIV. The timely affirmed serum hypo-osmolality and HN nadir are detectable before therapy only, and relate to severity [1].

$\mathrm{XV}$. The associated high sodium loss in hyperosmolar urine was documented in animal research [13] not in the post-TURP clinical research as bladder irrigation did not allow it.

XVI. The hydrocortisone replacement for SS HN covers its mineralocorticoid effect that prevents sodium loss contributing to the hyperosmolar urine.

XVII. Hydrocortisone is recommended for shock therapy- when used for combating the primary hypotension "episode" of the hyper-acute TURP syndrome HN shock it minimizes such sodium loss in urine. This partially explains the high sodium loss in hyper-osmolar urine during severe HN and hypoosmolality of the TUR syndrome.

XVIII. Excess sodium loss in urine despite serum hypoosmolality was detected in experimental setting by a great most prolific researcher on the TURP syndrome [13]. I thank the authors for resolving this conflicting issue.
XIX. The main cause of sodium loss in hyperosmolar urine despite serum hypo-osmolality is the excess $\mathrm{ADH}$ on the kidney. It implies predominance of volume over osmotic regulation by a responsive kidney. This may cause renal shut down or acute renal failure (ARF). So, VO/t may cause ARF as it does hypotension Shock!

XX. The rejuvenated hypertonic sodium therapy (HST) is the correct life-saving therapy of severe HN of "Critical" and "Close" to death Grades. It requires immediate rapid infusion of 5\% $\mathrm{NaCl}$ or $8.4 \% \mathrm{NaCO}$ - the later is readily available and equally effective.

XXI. Hypertonic Sodium is a potent ADH suppressor that induces massive dieresis with low sodium hypo-osmolar urinewhen high doses of loop diuretics fail [1]. By the end of 10-20 minutes infusion, recovery from coma is witnessed. It corrects the vascular hypotension shock and ARF- inducing massive dieresis shedding the excess VO. This dries the patient out of an internal drowning state, so please do not re-infuse similar volume again!

XXII. The hypertonic sodium therapy is no longer contraindicated, and was not an error when rejuvenated as anecdotal. It is the current therapy recommended by authorities on HN [14]. Its success has been proved by old reliable research $[3,4]$ as was rejuvenated and statistically proved in a recent prospective study [1]. It does miracle if timely given early before brain oedema causes permanent brain damage of coma and vegetative state [9-12], pontine myelinolysis [12] or other cerebral focal damage such as SS.

XXIII.Excess volumetric expansion by any infused VO2 fluid may mask or erase HN Nadir, cause dysnatraemia, hyperosmolarity gap and irreversible shock. Delay in therapy also causes permanent brain damage or death [8-12].

XXIV. Cranial nerves may be focally affected and total bilateral permanent blindness has been reported after the TURP procedure and brain oedema- with and without HN.

XXV. Even if all known causes of secondary HN are excluded, the prevalence of primary dilution $\mathrm{HN}$ in current hospital practice remains high ( $1 \%$ of all postoperative patients in USA [9]).

XXVI. The HN incidence of severe morbidity and mortality is also high. However, because it wears many presentation MASKs, rarely diagnosed at the hyper-acute setting, its serum HN Nadir can be easily erased or masked without being corrected on infusing VO2 fluid. Also because HN or the TURP syndrome is not listed as cause of death in any mortality register, the cause of death may be recorded under any name of its MASKs such as circulatory failure, cardiac, respiratory or cerebral infarction causes of death [5-12]. Thus the TURP syndrome is thought rare, not seen or does not exist.

XXVII. The TURP syndrome HN has apparently "vanished" from urology just by replacing VO1 (1.5\% Glycine) with VO2 
(Saline) as irrigating fluid for the TURP procedure! Or, is it reincarnating?

XXVIII. The incidence of HN in SS is 35\% in postpartum women $[15,16]$ and $16.8 \%$ in post trauma brain injury [17]. All cases have partial or complete empty Sella Turcica on MRI that, no doubt, will increasingly prove valuable tool in early diagnosis of VO/t insult and shock.

XXIX. The secondary HN of SS affects both women and men $[15,16]$. The TURP syndrome HN affects men and women after the TUR procedure, and affects men, women and children after $5 \%$ Glucose infusions [8-12]. The incidence of postoperative $\mathrm{HN}$ is $1 \%$ in USA [10].

XXX. Most VO/t goes missing from the overloaded circulation as it cannot be accommodated within the vascular system with maximum capacity of 7 litres and the excess far exceeds any loss by normally functioning kidneys before ARF occurs. Where does it all go?

XXXI.The intra-cellular part of the missing VO of both VO/t types causes primary brain oedema and/or pituitary infarctionthe exact pathological mechanism require verification.

XXXII. There are paradoxes here that are inconsistent with received wisdom and current recommendations based on accumulated errors. Examples: Hypotension is not synonymous with hypovolemia. Both types of $\mathrm{VO} / \mathrm{t}$ cause hypotension shock and ARF, but how and why? This require longer explanation and introduction of new evidence [1,2], but a short answer is:

XXXIII. A new phenomenon called magnetic hydrodynamic mixer discovered in the porous orifice $(G)$ tube [2] may prove powerful contender hypothesis for replacing the erroneous physiological low on the capillary-interstitial fluid exchange making the correct foundation for resolving $\mathrm{HN}$ and other puzzles of clinical syndromes caused by $\mathrm{VO} / \mathrm{t}$. The evidence on volumetric overload shocks (VOS) [18-20] and the replacement for Starling's law have been reported [21].

\section{References}

1. Ghanem AN, Ward JP (1990) Osmotic and metabolic sequelae of volumetric overload in relation to the TURP syndrome. Br J Uro 66(1): 71-78.

2. Ghanem AN (2001) Magnetic field-like fluid circulation of a porous orifice tube and its relevance to the capillary-interstitial fluid circulation: preliminary report. Medical Hypotheses 56(3): 325-334.

3. Harrison III RH, Boren JS, Robinson JR (1956) Dilutional hyponatraemic shock: another concept of the transurethral prostatic reaction. J Uro 75(1): 95-110.

4. Danowski TS, Winkler AW, Elkington JR (1946) The treatment of shock due to salt depression; comparison of isotonic, of hypertonic saline and of isotonic glucose solutions. J Clin Invest 25(1): 130-138.
5. Henderson DJ, Middleton RG (1980) Coma from hyponatraemia of the transurethral resection of prostate. Urology 15(3): 267-271.

6. Spencer Hoyt H, Goebel J L, Lee HI, Schoenbrod J (1958) Types of shock reaction during transurethral resection and relation to acute renal failure. J Uro 79(3): 500-507.

7. Charlton AJ (1980) Cardiac arrest during transurethral surgery after absorption of 1.5\% glycine. Anaesth 35: 804-807.

8. Heytens L, Camu F (1984) Pulmonary edema during caesarean section related to the use of oxytocin drugs. Acta Anaesthesiologica Belgica 35(2): 155-164.

9. Arieff AI (1986) Hyponatraemia, convulsion, respiratory arrest and permanent brain damage after elective surgery in healthy women. N Engl J Med 314(24): 1529-1534.

10. Ayus JC (1993) Endometrial ablation complicated by fatal hyponatraemic encephalopathy. JAMA 270(10): 1230-1232.

11. Arieff AI (1992) Hyponatraemia and death or permanent brain damage in healthy children. Br Med Jour 304(6836): 1218-1222.

12. Thompson PD, Gledhill RF, Quinn NP, Rossor MN, Stanley P, et al. (1986) Neurological complications associated with parentral treatment: central pontine myelinolysis and Wernicke's encephalopathy. Br Med Jour 292(6521): 684-685.

13. Hahn RG (1997) Irrigating fluids in endoscopic surgery. Br J Uro 79: 669680 .

14. Arieff AI (1993) Management of hyponatraemia. Br Med Jour 307(6899): 305-308.

15. Toshimitsu Yatagai, Kusaka I, Nakamura T, Nagasaka S, Honda K, et al. (2003) Close association of severe hyponatremia with exaggerated release of arginine vasopressin in elderly subjects with secondary adrenal insufficiency. European Journal of Endocrinology 148(2): 221226.

16. Dökmeta HS, Kilicli F, Korkmaz S, Yonem O (2006) Characteristic features of 20 patients with Sheehan's syndrome. Gynecological. Endocrinology 22(5): 279-283.

17. Nobuhiro Moro, Katayama Y, Igarashi T, Mori T, Kawamata T, et al. (2007) Hyponatremia in patients with traumatic brain injury: incidence, mechanism, and response to sodium supplementation or retention therapy with hydrocortisone. Surgical Neurology 68(4): 387-393.

18. Ghanem AN, Ghanem SA (2016) Volumetric Overload Shocks: Why Is Starling's Law for Capillary Interstitial Fluid Transfer Wrong? The Hydrodynamics of a Porous Orifice Tube as Alternative. Surgical Science $7(6): 245-249$.

19. Pindoria N, Ghanem SA, Ghanem KA, Ghanem AN (2017) Volumetric overload shocks in the patho-etiology of the transurethral resection prostatectomy syndrome and acute dilution hyponatraemia. Integr Mol Med.

20. Ghanem KA, Ghanem AN (2017) Volumetric overload shocks in the patho-etiology of the transurethral resection prostatectomy syndrome and acute dilution hyponatraemia: The clinical evidence based on 23 case series. Basic Research Journal of Medicine and Clinical Sciences 6(4): $35-43$.

21. Ghanem KA, Ghanem AN (2017) The proof and reasons that Starling's law for the capillary-interstitial fluid transfer is wrong, advancing the hydrodynamics of a porous orifice (G) tube as the real mechanism. Blood, Heart and Circ 1(1): 1-7. 


\section{ISSN: 2574-1241}

DOI: $10.26717 / B J S T R .2018 .11 .002130$

Ahmed N Ghanem. Biomed J Sci \& Tech Res

(C) This work is licensed under Creative

Submission Link: https://biomedres.us/submit-manuscript.php

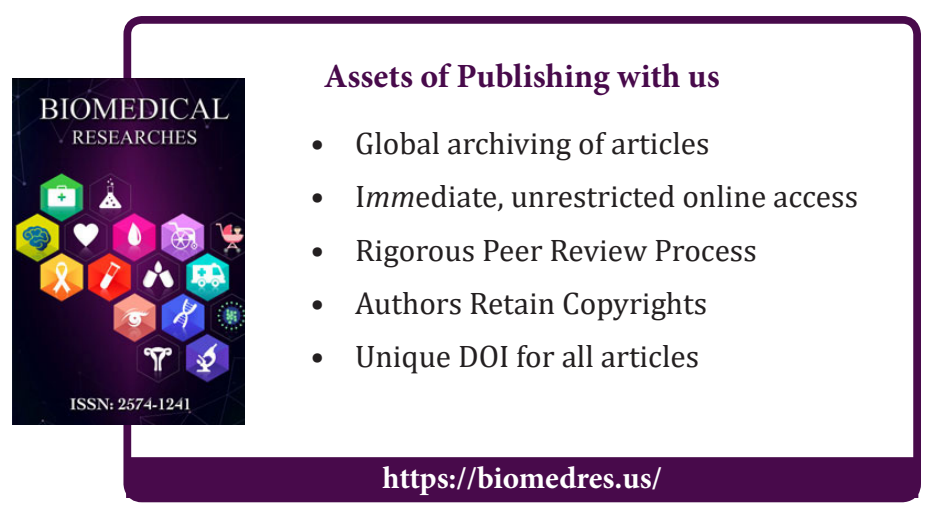

